\title{
A Convenient Preparation of Bis(phosphorothioyl) Sulfides
}

Shi Min, Hideharu Ishihara, Toshiaki Murai, and Shinzi Kato*

Department of Chemistry, Faculty of Engineering, Gifu University,

Yanagido, Gifu 501-11, Japan

Z. Naturforsch. 44b, 153-157 (1989); received September 23, 1988

Bis(phosphorothioyl) Sulfides, Piperidinium Phosphorodithioates, Bis(phosphorothioyl) Oxides

The reaction of piperidinium and potassium phosphorodithioates with 2-chloro-1-methylpyridinium salts afforded the corresponding bis(phosphorothioyl) sulfides in good yields. The similar reaction with potassium diphenylphosphorothioate gave exclusively bis(diphenylphosphorothioyl) oxide.

\section{Introduction}

In general, bis(phosphorothioyl) sulfides (3) which are effective as fungicides [1,2], cannot be obtained from $\mathrm{O}, \mathrm{O}^{\prime}$-dialkyl or diarylphosphorodithioic acid salts with phosphorochloridothioate [3]. Therefore, several methods have been proposed for the preparation of 3 [4-9], and the following two methods have been employed mainly: a) Reaction of phosphorodithioic acid with aminosulfenyl chloride [5]. b) Desulfurization of bis(phosphorothioyl) disulfides by phosphine [6] or phosphites [8]. These methods, however, have some disadvantages such as limited availability of the starting aminosulfenyl chlorides or the difficult purification of the product 3. Recently, we have found that piperidinium dithiocarboxylates readily react with 2-chloro-1-methylpyridinium salts to give the corresponding bis(thioacyl) sulfides in good yields [10]. This result stimulated us to develop an alternative, convenient preparation of the title compounds 3 from the reaction of piperidinium phosphorodithioates (1) with 2-chloro-1-methylpiperidinium salts (2).

\section{Results and Discussion}

When 2-chloro-1-methylpyridinium $p$-toluenesulfonate was added to a solution of piperidinium $\mathrm{O}, \mathrm{O}^{\prime}$ diphenylphosphorodithioate (1f) in dichloromethane, the reaction mixture gradually changed from colorless to yellow. After stirring for $1 \mathrm{~h}$, usual workup of the mixture and then chromatographic separation gave bis $\left(\mathrm{O}, \mathrm{O}^{\prime}\right.$-diphenylphosphinothioyl $)$ sulfide (3f) in $66 \%$ yield as colorless crystals. The

\footnotetext{
* Reprint requests to Dr. S. Kato.

Verlag der Zeitschrift für Naturforschung, D-7400 Tübingen 0932-0776/89/0200-0153/\$ 01.00/0
}

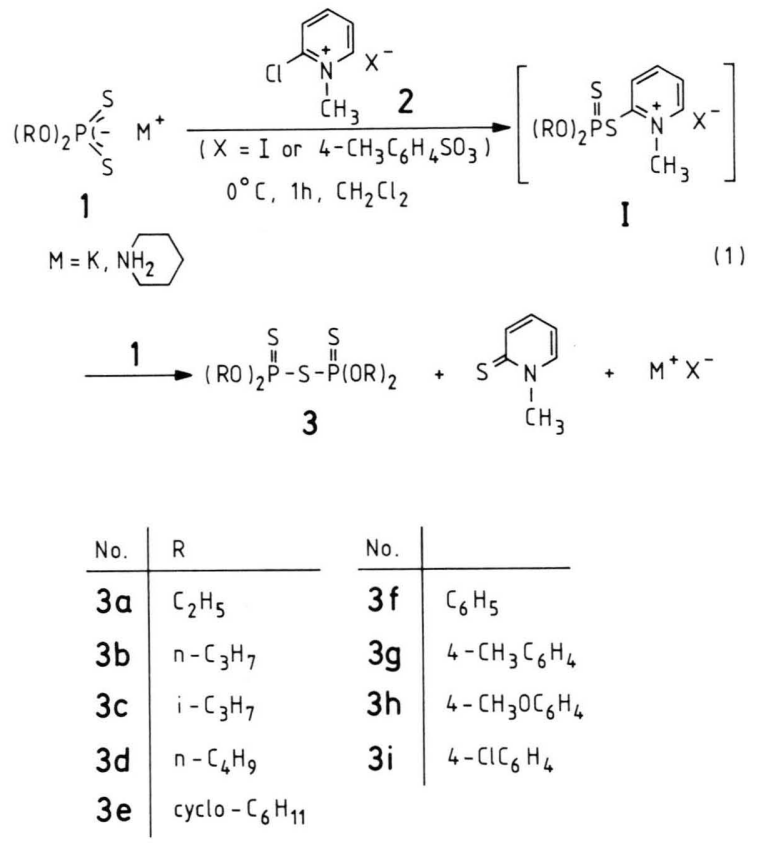

reactions with other aliphatic and aromatic phosphorodithioates $(\mathbf{1} \mathbf{a}-\mathbf{e}, \mathbf{g}-\mathbf{i})$ under the same conditions yielded $60-80 \%$ of the corresponding bis(phosphorothioyl) sulfides $(\mathbf{3 a}-\mathbf{e}, \mathbf{g}-\mathbf{i})$. Similar reactions with $\mathrm{O}, \mathrm{O}^{\prime}$-diphenylphosphinodithioic acid potassium salts instead of the piperidinium salts (1f) or with the iodides $(\mathbf{2}, \mathrm{X}=\mathrm{I})$ instead of the $p$-toluenesulfonate provided analogous yields of $\mathbf{3}$, while the use of the silver and zinc salts led to 5 and $10 \%$, respectively, because of their low solubility and/or reduced reactivity.

It is noted that the reaction of piperidinium diphenylphosphinothioate (7) with 2 afforded $40 \%$ of bis(diphenylphosphinothioyl) oxide (8) (eq. (2)). 


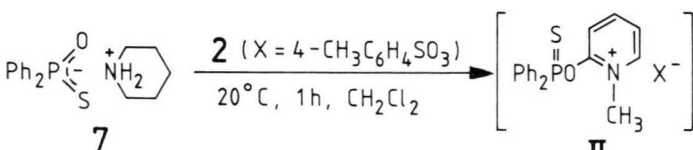

II

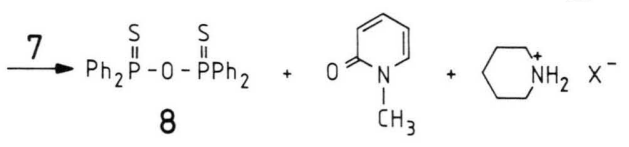

9

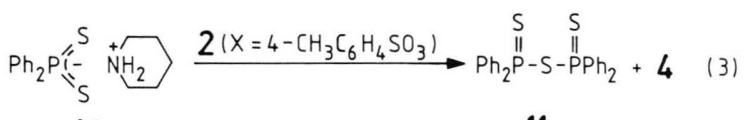

10

11

No formation of the possible diphenylphosphinothioyl sulfide (10) or bis(diphenylphosphinyl) sulfide (11) was detected. The reaction would proceed via the intermediate II, wich is attacked by the oxygen atom of diphenylphosphinothioate anion (7).

The structures of the products $\mathbf{3}$ and $\mathbf{8}$ were established by spectral data and microanalysis and/or by comparison with authentic samples.

\section{Experimental}

Melting points were determined using a Yanagimoto micro melting point apparatus and are uncorrected. The IR spectra were measured on a JASCO grating IR spectrometer IR-G. The ${ }^{1} \mathrm{H}$ and ${ }^{31} \mathrm{P}$ NMR spectra were recorded on Hitachi R-20 $(90 \mathrm{MHz})$ and JEOL-JNM-GX270 (270 MHz) with tetramethylsilane as internal standard. Mass spectra were taken by Hitachi RMN-6M mass spectrometer at an ionizing voltage of $20 \mathrm{eV}$. Elemental analyses were performed by the Elemental Analysis Center of Kyoto University.

\section{Materials}

Piperidinium $\mathrm{O}, \mathrm{O}^{\prime}$-diaralkylphosphorodithioates (1) except for the $\mathrm{O}, \mathrm{O}^{\prime}$-diethyl derivative (1a) [12] were prepared by slight modification of the method described in literature [13]. Their yields and physical properties are summarized in Table II. Potassium [14], silver [15] and zinc $\mathrm{O}, \mathrm{O}^{\prime}$-diphenylphosphorodithioates [16], and potassium diphenylphosphinothioate [17] and diphenylphosphinodithioate [18] were prepared by the reaction of the corresponding phosphorothioic and -dithioic acids with

Table I. Yields and physical properties of bis(phosphorothioyl) sulfides (3).

\begin{tabular}{|c|c|c|c|c|c|c|c|}
\hline No. & $\begin{array}{l}\stackrel{\mathrm{S}}{\|} \underset{\mathrm{R}}{\|} \stackrel{\mathrm{S}}{\|})_{2} \mathrm{P}-\mathrm{S}-\mathrm{P}(\mathrm{OR})_{2} \\
\end{array}$ & $\begin{array}{l}\text { Yield } \\
{[\%]}\end{array}$ & $\begin{array}{l}\text { m.p. } \\
{\left[{ }^{\circ} \mathrm{C}\right]}\end{array}$ & $\begin{array}{l}\mathrm{IR}(\mathrm{KBr}) \\
\nu \mathrm{P}=\mathrm{S}\left[\mathrm{cm}^{-1}\right]\end{array}$ & $\begin{array}{l}\operatorname{Mass}^{\mathrm{a}} \\
\mathrm{m} / \mathrm{z}\end{array}$ & $\begin{array}{l}{ }^{31} \mathrm{P} \mathrm{NMR}^{\mathrm{b}} \\
{[\delta]}\end{array}$ & $\begin{array}{l}{ }^{1} \mathrm{H} \text { NMR }\left(\mathrm{CDCl}_{3}\right) \\
{[\delta]}\end{array}$ \\
\hline $3 \mathbf{a}$ & $\mathrm{C}_{2} \mathrm{H}_{5}$ & 77 & $70-72$ & 650 & 338 & +78.4 & $\begin{array}{l}1.35\left(\mathrm{t}, 3 \mathrm{H}, \mathrm{CH}_{3}\right), 4.00(\mathrm{qd} \\
\left.J_{\mathrm{POCH}}=10 \mathrm{~Hz}, 2 \mathrm{H}, \mathrm{CH}_{2}\right)\end{array}$ \\
\hline $3 \mathbf{b}$ & $\mathrm{C}_{3} \mathrm{H}_{7}$ & 65 & oil & $675^{\mathrm{c}}$ & 394 & +77.2 & $\begin{array}{l}0.96\left(\mathrm{t}, 3 \mathrm{H}, \mathrm{CH}_{3}\right), 1.7\left(\mathrm{~m}, 2 \mathrm{H}, \mathrm{CH}_{2}\right), \\
3.98\left(\mathrm{td}, J_{\mathrm{POCH}}=9 \mathrm{~Hz}, 2 \mathrm{H}, \mathrm{CH}_{2} \mathrm{O}\right)\end{array}$ \\
\hline $3 \mathbf{c}$ & $i-\mathrm{C}_{3} \mathrm{H}_{7}$ & 61 & oil & $635^{\mathrm{c}}$ & 394 & +76.0 & $\begin{array}{l}1.40\left(\mathrm{~d}, 6 \mathrm{H}, \mathrm{CH}_{3}\right), 4.83(\mathrm{~m}, \\
\left.J_{\mathrm{POCH}}=10 \mathrm{~Hz}, 1 \mathrm{H}, \mathrm{CHO}\right)\end{array}$ \\
\hline 3d & $\mathrm{C}_{4} \mathrm{H}_{9}$ & 63 & oil & $645^{c}$ & 450 & +77.2 & $\begin{array}{l}0.91\left(\mathrm{t}, 3 \mathrm{H}, \mathrm{CH}_{3}\right), 1.2-1.8(\mathrm{~m}, 4 \mathrm{H}, \\
\left.\mathrm{CH}_{2}\right), 4.10\left(\mathrm{br}, 2 \mathrm{H}, \mathrm{CH}_{2} \mathrm{O}\right)\end{array}$ \\
\hline $3 \mathbf{e}$ & cyclo- $\mathrm{C}_{6} \mathrm{H}_{11}$ & 86 & oil & $660^{c}$ & 554 & +76.3 & $\begin{array}{l}1.5 \text { and } 1.8\left(\mathrm{br}, 10 \mathrm{H}, \mathrm{CH}_{2}\right) \\
4.6(\mathrm{~m}, 1 \mathrm{H}, \mathrm{CHO})\end{array}$ \\
\hline $3 \mathbf{f}$ & $\mathrm{C}_{6} \mathrm{H}_{5}$ & $\begin{array}{l}66 \\
60^{\mathrm{d}} \\
5^{\mathrm{e}} \\
10^{\mathrm{f}}\end{array}$ & $131-133$ & 645 & 530 & +70.3 & $7.1-7.4(\mathrm{~m}, \mathrm{Ar})$ \\
\hline $3 \mathbf{g}$ & $3-\mathrm{CH}_{3} \mathrm{C}_{6} \mathrm{H}_{4}$ & 54 & oil & $645^{c}$ & 586 & +70.4 & $\begin{array}{l}2.28\left(\mathrm{~s}, 3 \mathrm{H}, \mathrm{CH}_{3}\right), 6.70-7.40(\mathrm{~m}, \\
4 \mathrm{H}, \mathrm{Ar})\end{array}$ \\
\hline $3 \mathbf{h}$ & $4-\mathrm{CH}_{3} \mathrm{C}_{6} \mathrm{H}_{4}$ & 59 & $91-93$ & 645 & 586 & +71.4 & $2.28\left(\mathrm{~s}, 3 \mathrm{H}, \mathrm{CH}_{3}\right), 7.12$ (bs, $\left.4 \mathrm{H}, \mathrm{Ar}\right)$ \\
\hline $3 \mathbf{i}$ & $2-\mathrm{CH}_{3} \mathrm{OC}_{6} \mathrm{H}_{4}$ & 30 & oil & $640^{c}$ & 650 & +71.8 & $\begin{array}{l}3.71\left(\mathrm{~s}, 3 \mathrm{H}, \mathrm{CH}_{3} \mathrm{O}\right), 6.7-7.0(\mathrm{~m}, \\
4 \mathrm{H}, \mathrm{Ar})\end{array}$ \\
\hline $3 \mathbf{j}$ & $4-\mathrm{CH}_{3} \mathrm{OC}_{6} \mathrm{H}_{4}$ & 75 & $146-148$ & 640 & 650 & +72.9 & $\begin{array}{l}3.78\left(\mathrm{~s}, 3 \mathrm{H}, \mathrm{CH}_{3}\right), 6.80(\mathrm{~d}, 2 \mathrm{H}, \mathrm{Ar}), \\
7.18(\mathrm{~d}, 2 \mathrm{H}, \mathrm{Ar})\end{array}$ \\
\hline $3 \mathbf{k}$ & $4-\mathrm{ClC}_{6} \mathrm{H}_{4}$ & 57 & $74-76$ & 642 & 666 & +70.1 & $6.75(\mathrm{~d}, 2 \mathrm{H}, \mathrm{Ar}), 7.13(\mathrm{~d}, 2 \mathrm{H}, \mathrm{Ar})$ \\
\hline
\end{tabular}

a $20 \mathrm{eV}, 110{ }^{\circ} \mathrm{C}$; ${ }^{\mathrm{b}}$ reference $85 \% \mathrm{H}_{3} \mathrm{PO}_{4}$ (slovent: $\mathrm{CH}_{2} \mathrm{Cl}_{2}$ ); ${ }^{\mathrm{c}}$ neat; ${ }^{\mathrm{d}}$ using potassium $\mathrm{O}, \mathrm{O}^{\prime}$-diphenylphosphinodithioate: ${ }^{\mathrm{e}}$ using zinc bis $\left(\mathrm{O}, \mathrm{O}^{\prime}\right.$-diphenylphosphinodithioate $)$; ${ }^{\mathrm{f}}$ using silver $\mathrm{O}, \mathrm{O}^{\prime}$-diphenylphosphinodithioate. 
potassium bicarbonate, silver nitrate, or with zinc diacetate. 2-Chloro-1-methylpyridinium iodide and $p$-toluenesulfonate were commerical grade and used without further purification.

The preparations of bis $\left(\mathrm{O}, \mathrm{O}^{\prime}\right.$-diethylphosphorothioyl) (3a) and $\operatorname{bis}\left(\mathrm{O}, \mathrm{O}^{\prime}\right.$-di- $p$-tolyl(phosphorothioyl)) sulfides $(\mathbf{3} \mathbf{h})$ are described in detail as typical procedure for the preparation of bis(phosphorothioyl) sulfides 3 . The physical properties of $\mathbf{3}$ are summarized in Table I.

\section{Bis $\left(O, O^{\prime}\right.$-diethylphosphorothioyl) sulfide (3a)}

2-Chloro-1-methylpyridinium $p$-toluenesulfonate (150 mg, $0.5 \mathrm{mmol}$ ) was added to a solution of piperidinium $\mathrm{O}, \mathrm{O}^{\prime}$-diethylphosphorodithioate (1a) $(271 \mathrm{mg}, 1 \mathrm{mmol})$ in dichloromethane $(10 \mathrm{ml})$, and the reaction mixture was stirred at $30^{\circ} \mathrm{C}$ for $1 \mathrm{~h}$. After removal of the solvent by rotary evaporator, thin layer chromatography (TCL) of the residue [dichloromethane/hexane $\left.(1: 5), \quad R_{f}=0.60\right]$, gave
$130 \mathrm{mg}(77 \%)$ of $\mathbf{3 a}$ as colorless crystals; m.p. $70-72{ }^{\circ} \mathrm{C}$.

\section{Bis $\left(O, O^{\prime}\right.$-di-propylphosphorothioyl) sulfide (3) $\left.\mathbf{3}\right)$}

The reaction of 2-chloro-1-methylpyridinium $p$-toluenesulfonate $(150 \mathrm{mg}, 0.5 \mathrm{mmol})$ with piperidinium $\mathrm{O}, \mathrm{O}^{\prime}$-di-propylphosphorodithioate $(\mathbf{1} \mathbf{b})$ $(299 \mathrm{mg}, 1 \mathrm{mmol})$, followed by TLC [dichloromethane/hexane $\left.(1: 2), R_{f}=0.51\right]$, gave $128 \mathrm{mg}$ $(65 \%)$ of $\mathbf{3 b}$ as a slightly yellow oil. Exact mass (70 eV) calcd for $\mathrm{C}_{12} \mathrm{H}_{28} \mathrm{O}_{4} \mathrm{P}_{2} \mathrm{~S}_{3} ; \mathrm{m} / z$ 394.0624; found 394.0629 .

\section{$\operatorname{Bis}\left(O, O^{\prime}\right.$-di-isopropylphosphorothioyl) sulfide (3c)}

The reaction of 2-chloro-1-methylpyridinium $p$-toluenesulfonate $(150 \mathrm{mg}, 0.5 \mathrm{mmol})$ with piperidinium $\mathrm{O}, \mathrm{O}^{\prime}$-di-isopropylphosphorodithioate (1c) (299 mg, $1 \mathrm{mmol}$ ), followed by TLC [ethyl acetate/ hexane (1:7), $\left.R_{f}=0.75\right]$, gave $120 \mathrm{mg}(61 \%)$ of $\mathbf{3 c}$ as a slightly yellow oil.

Table II. Yields and physical properties of piperidinium phosphorodithioates (1).

\begin{tabular}{|c|c|c|c|c|c|c|}
\hline No. & $\begin{array}{l}\text { Compounds } \\
\mathrm{R}\end{array}$ & $\begin{array}{l}\text { Yield } \\
{[\%]}\end{array}$ & $\begin{array}{l}\mathrm{m} \cdot \mathrm{p} . \\
{\left[{ }^{\circ} \mathrm{C}\right]}\end{array}$ & $\begin{array}{l}\mathrm{IR}(\mathrm{KBr}) \\
v \mathrm{PS}_{2}\left[\mathrm{~cm}^{-1}\right]\end{array}$ & $\begin{array}{l}{ }^{1} \mathrm{H} \text { NMR }\left(\mathrm{CDCl}_{3}\right) \\
{[\delta]}\end{array}$ & $\begin{array}{l}\text { Recryst. } \\
\text { solvent }\end{array}$ \\
\hline $1 \mathbf{a}$ & $\mathrm{C}_{2} \mathrm{H}_{5}$ & 73 & $\begin{array}{l}55-57 \\
57[12]\end{array}$ & 825,670 & $\begin{array}{l}1.30\left(\mathrm{t}, 6 \mathrm{H}, \mathrm{CH}_{3}\right), 1.5-2.0\left(\mathrm{br}, 6 \mathrm{H}, \mathrm{CH}_{2}\right), \\
3.23\left(\mathrm{t}, 4 \mathrm{H}, \mathrm{CH}_{2} \mathrm{~N}\right), 4.03\left(\mathrm{qd}, J_{\mathrm{POCH}}=10 \mathrm{~Hz}, 4 \mathrm{H},\right. \\
\left.\mathrm{CH}_{2}\right), 7.5\left(\mathrm{bs}, 2 \mathrm{H},{ }^{+} \mathrm{NH}_{2}\right)\end{array}$ & $\mathrm{Et}_{2} \mathrm{O}$ \\
\hline $1 \mathbf{b}$ & $\mathrm{C}_{3} \mathrm{H}_{7}$ & 87 & $50-52$ & 825,670 & $\begin{array}{l}0.98\left(\mathrm{t}, 6 \mathrm{H}, \mathrm{CH}_{3}\right), 1.5-2.0\left(\mathrm{~m}, 10 \mathrm{H}, \mathrm{CH}_{2}\right) \\
3.3\left(\mathrm{~b}, 4 \mathrm{H}, \mathrm{CH}_{2} \mathrm{~N}\right), 3.87\left(\mathrm{td}, J_{\mathrm{POCH}}=9 \mathrm{~Hz}, 4 \mathrm{H}\right. \\
\left.\mathrm{CH}_{2} \mathrm{O}\right), 8.6\left(\mathrm{~b}, 2 \mathrm{H},{ }^{+} \mathrm{NH}_{2}\right)\end{array}$ & $\mathrm{Et}_{2} \mathrm{O}$ \\
\hline $1 \mathrm{c}$ & $i-\mathrm{C}_{3} \mathrm{H}_{7}$ & 84 & $114-116$ & 815,660 & $\begin{array}{l}1.28\left(\mathrm{~d}, 12 \mathrm{H}, \mathrm{CH}_{3}\right), 1.40-2.10\left(\mathrm{~m}, 6 \mathrm{H}, \mathrm{CH}_{2}\right), \\
3.32\left(\mathrm{t}, 4 \mathrm{H}, \mathrm{CH}_{2} \mathrm{~N}\right), 4.7\left(\mathrm{~m}, J_{\mathrm{POCH}}=10 \mathrm{~Hz}, 2 \mathrm{H},\right. \\
\mathrm{CHO}), 7.55\left(\mathrm{br}, 2 \mathrm{H},{ }^{+} \mathrm{NH}_{2}\right)\end{array}$ & $\mathrm{AcOEt}$ \\
\hline $1 d$ & $\mathrm{C}_{4} \mathrm{H}_{9}$ & 68 & $62-63$ & 815,670 & $\begin{array}{l}0.85\left(\mathrm{t}, 6 \mathrm{H}, \mathrm{CH}_{3}\right), 1.2-1.8\left(\mathrm{~m}, 14 \mathrm{H}, \mathrm{CH}_{2}\right), \\
3.1\left(\mathrm{br}, 4 \mathrm{H}, \mathrm{CH}_{2} \mathrm{~N}\right), 3.95\left(\mathrm{td}, J_{\mathrm{POCH}}=9 \mathrm{~Hz}, 4 \mathrm{H},\right. \\
\left.\mathrm{CH}_{2} \mathrm{O}\right), 8.7\left(\mathrm{br}, 2 \mathrm{H},{ }^{+} \mathrm{NH}_{2}\right)\end{array}$ & $\begin{array}{l}\mathrm{CH}_{2} \mathrm{Cl}_{2} / \mathrm{C}_{6} \mathrm{H}_{14} \\
(4: 1)\end{array}$ \\
\hline $1 \mathbf{e}$ & cyclo- $\mathrm{C}_{6} \mathrm{H}_{11}$ & 65 & $145-147$ & 809,665 & $\begin{array}{l}1.2-2.2\left(\mathrm{br}, 26 \mathrm{H}, \mathrm{CH}_{2}\right), 3.35\left(\mathrm{br}, 4 \mathrm{H}, \mathrm{CH}_{2} \mathrm{~N}\right) \\
4.4(\mathrm{br}, 2 \mathrm{H}, \mathrm{CHO}), 8.8\left(\mathrm{br}, 2 \mathrm{H},{ }^{+} \mathrm{NH}_{2}\right)\end{array}$ & $\begin{array}{l}\mathrm{CH}_{2} \mathrm{Cl}_{2} / \mathrm{C}_{6} \mathrm{H}_{14} \\
(6: 1)\end{array}$ \\
\hline $1 \mathrm{f}$ & $\mathrm{C}_{6} \mathrm{H}_{5}$ & 77 & $113-115$ & 815,655 & $\begin{array}{l}1.4-2.0\left(\mathrm{br}, 6 \mathrm{H}, \mathrm{CH}_{2}\right), 3.0\left(\mathrm{br}, 4 \mathrm{H}, \mathrm{CH}_{2} \mathrm{~N}\right) \\
7.0-7.5(\mathrm{~m}, 10 \mathrm{H}, \mathrm{Ar}), 8.12\left(\mathrm{br}, 2 \mathrm{H},{ }^{+} \mathrm{NH}_{2}\right)\end{array}$ & $\begin{array}{l}\mathrm{CH}_{2} \mathrm{Cl}_{2} / \mathrm{C}_{6} \mathrm{H}_{14} \\
(6: 1)\end{array}$ \\
\hline $1 \mathrm{~g}$ & $3-\mathrm{CH}_{3} \mathrm{C}_{6} \mathrm{H}_{4}$ & 80 & $124-127$ & 800,635 & $\begin{array}{l}1.3-1.8\left(\mathrm{~m}, 6 \mathrm{H}, \mathrm{CH}_{2}\right), 2.30\left(\mathrm{~s}, 6 \mathrm{H}, \mathrm{CH}_{3}\right), \\
2.95\left(\mathrm{br}, 4 \mathrm{H}, \mathrm{CH}_{2} \mathrm{~N}\right), 6.7-6.9 \text { and } 7.0-7.1(\mathrm{~m}, \\
8 \mathrm{H}, \mathrm{Ar}), 8.0\left(\mathrm{~b}, 2 \mathrm{H},{ }^{+} \mathrm{NH}_{2}\right)\end{array}$ & $\begin{array}{l}\mathrm{CH}_{2} \mathrm{Cl}_{2} / \mathrm{C}_{6} \mathrm{H}_{14} \\
(6: 1)\end{array}$ \\
\hline $1 \mathrm{~h}$ & $4-\mathrm{CH}_{3} \mathrm{C}_{6} \mathrm{H}_{4}$ & 73 & $138-140$ & 800,640 & $\begin{array}{l}1.3-1.8\left(\mathrm{~m}, 6 \mathrm{H}, \mathrm{CH}_{2}\right), 2.38\left(\mathrm{~s}, 6 \mathrm{H}, \mathrm{CH}_{3}\right) \\
2.9\left(\mathrm{br}, 4 \mathrm{H}, \mathrm{CH}_{2} \mathrm{~N}\right), 6.9-7.3(\mathrm{~m}, 8 \mathrm{H}, \mathrm{Ar}) \\
8.1\left(\mathrm{~b}, 2 \mathrm{H},{ }^{+} \mathrm{NH}_{2}\right)\end{array}$ & $\begin{array}{l}\mathrm{CH}_{2} \mathrm{Cl}_{2} / \mathrm{C}_{6} \mathrm{H}_{14} \\
(6: 1)\end{array}$ \\
\hline $1 \mathbf{i}$ & $2-\mathrm{CH}_{3} \mathrm{OC}_{6} \mathrm{H}_{4}$ & 80 & $157-159$ & 810,630 & $\begin{array}{l}1.0-2.0\left(\mathrm{~m}, 6 \mathrm{H}, \mathrm{CH}_{2}\right), 3.20\left(\mathrm{br}, 4 \mathrm{H}, \mathrm{CH}_{2} \mathrm{~N}\right) \\
3.78\left(\mathrm{~s}, 6 \mathrm{H}, \mathrm{CH}_{3} \mathrm{O}\right), 6.5-7.1 \text { and } 7.3-7.4(\mathrm{~m}, \\
8 \mathrm{H}, \mathrm{Ar}), 8.0\left(\mathrm{br}, 2 \mathrm{H},{ }^{+} \mathrm{NH}_{2}\right)\end{array}$ & $\begin{array}{l}\mathrm{CH}_{2} \mathrm{Cl}_{2} / \mathrm{C}_{6} \mathrm{H}_{14} \\
(7: 1)\end{array}$ \\
\hline $\mathbf{1 j}$ & $4-\mathrm{CH}_{3} \mathrm{OC}_{6} \mathrm{H}_{4}$ & 76 & $93-95$ & 800,640 & $\begin{array}{l}1.2-2.0\left(\mathrm{~m}, 6 \mathrm{H}, \mathrm{CH}_{2}\right), 3.0\left(\mathrm{br}, 4 \mathrm{H}, \mathrm{CH}_{2} \mathrm{~N}\right) \\
3.78\left(\mathrm{~s}, 6 \mathrm{H}, \mathrm{CH}_{3} \mathrm{O}\right), 6.82(\mathrm{~d}, 4 \mathrm{H}, \mathrm{Ar}), 7.27(\mathrm{~d}, \\
4 \mathrm{H}, \mathrm{Ar}), 8.1\left(\mathrm{br}, 2 \mathrm{H},{ }^{+} \mathrm{NH}_{2}\right)\end{array}$ & $\begin{array}{l}\mathrm{CH}_{2} \mathrm{Cl}_{2} / \mathrm{C}_{6} \mathrm{H}_{14} \\
(6: 1)\end{array}$ \\
\hline $1 \mathbf{k}$ & $4-\mathrm{ClC}_{6} \mathrm{H}_{4}$ & 71 & $106-108$ & 810,630 & $\begin{array}{l}1.3-1.8(\mathrm{~m}, 6 \mathrm{H}, 2 \mathrm{H}), 2.7-3.1\left(\mathrm{~m}, 4 \mathrm{H}, \mathrm{CH}_{2} \mathrm{~N}\right) \\
7.12(\mathrm{~s}, 8 \mathrm{H}, \mathrm{Ar}), 8.0\left(\mathrm{br}, 2 \mathrm{H},{ }^{+} \mathrm{NH}_{2}\right)\end{array}$ & $\begin{array}{l}\mathrm{CH}_{2} \mathrm{Cl}_{2} / \mathrm{C}_{6} \mathrm{H}_{14} \\
(6: 1)\end{array}$ \\
\hline
\end{tabular}

Reaction conditions: $1 \mathbf{a}-\mathbf{e}=40^{\circ} \mathrm{C}, 3 \mathrm{~h} ; \mathbf{1 f}-\mathbf{k}=150{ }^{\circ} \mathrm{C}, 3 \mathrm{~h}$. 


\section{$\operatorname{Bis}\left(O, O^{\prime}\right.$-di-butylphosphorothioyl) sulfide (3d)}

The reaction of 2-chloro-1-methylpyridinium $p$-toluenesulfonate $(150 \mathrm{mg}, 0.5 \mathrm{mmol})$ with piperidinium $\mathrm{O}, \mathrm{O}^{\prime}$-di-butylphosphorodithioate (1d) (327 mg, $1 \mathrm{mmol}$ ), followed by TLC [ethyl acetate/ hexane (1:7), $\left.R_{f}=0.80\right]$, gave $142 \mathrm{mg}(63 \%)$ of $\mathbf{3 d}$ as a slightly yellow oil.

\section{$\operatorname{Bis}\left(O, O^{\prime}\right.$-di-cyclohexylphosphorothioyl) sulfide (3e)}

The reaction of 2-chloro-1-methylpyridinium $p$-toluenesulfonate $(150 \mathrm{mg}, \quad 0.5 \mathrm{mmol})$ with piperidinium $\mathrm{O}, \mathrm{O}^{\prime}$-di-cyclohexylphosphorodithioate $(\mathbf{1} \mathbf{e})$ (379 mg, $1 \mathrm{mmol}$ ), followed by TLC [ethyl acetate/ hexane $\left.(1: 5), R_{f}=0.80\right]$, gave $188 \mathrm{mg}(68 \%)$ of $\mathbf{3 e}$ as a slightly yellow oil. Exact mass $(70 \mathrm{eV})$ : calcd for $\mathrm{C}_{24} \mathrm{H}_{20} \mathrm{O}_{4} \mathrm{P}_{2} \mathrm{~S}_{3} ; m / z$ 554.1875; found 554.1891.

\section{Bis $\left(O, O^{\prime}\right.$-diphenylphosphorothioyl) sulfide (3f)}

The reaction of 2-chloro-1-methylpyridinium $p$-toluenesulfonate $(150 \mathrm{mg}, 0.5 \mathrm{mmol})$ with piperidinium $\mathrm{O}, \mathrm{O}^{\prime}$-diphenylphosphorodithioate (1f) (367 mg, $1 \mathrm{mmol}$ ), followed by TLC [ethyl acetate/ hexane $\left.(1: 5), R_{f}=0.48\right]$ and by recrystallization from dichloromethane/hexane (1:3), gave $175 \mathrm{mg}$ $(66 \%)$ of $\mathbf{3 f}$ as colorless crystals.

$$
\begin{aligned}
& \mathrm{C}_{24} \mathrm{H}_{10} \mathrm{P}_{2} \mathrm{O}_{4} \mathrm{~S}_{3} \text { (553.55) } \\
& \text { Found C 53.92 H 3.72, } \\
& \text { Calcd C } 54.33 \text { H } 3.80 \text {. }
\end{aligned}
$$

The reaction with potassium $\mathrm{O}, \mathrm{O}^{\prime}$-diphenylphosphorodithioate $(320 \mathrm{mg}, 1 \mathrm{mmol})$ gave $320 \mathrm{mg}$ $(60 \%)$ of $\mathbf{3 f}$.

The reaction with silver $\mathrm{O}, \mathrm{O}^{\prime}$-diphenylphosphorodithioate) $(389 \mathrm{mg}, 1 \mathrm{mmol})$ in THF at $66^{\circ} \mathrm{C}$ for $1 \mathrm{~h}$ gave $27 \mathrm{mg}(10 \%)$ of $\mathbf{3 f}$ and $76 \%$ of the starting silver salt.

The reaction with zinc bis $\left(\mathrm{O}, \mathrm{O}^{\prime}\right.$-diphenylphosphorodithioate) $(314 \mathrm{mg}, 1 \mathrm{mmol})$ in THF at $66^{\circ} \mathrm{C}$ for $1 \mathrm{~h}$ gave $13 \mathrm{mg} \mathrm{(5 \% )}$ of $\mathbf{3 f}$ and $84 \%$ of the starting zinc salt.

\section{Bis $\left(O, O^{\prime}\right.$-di-m-tolylphosphorothioyl) sulfide $(\mathbf{3} \mathbf{g})$}

The reaction of 2-chloro-1-methylpyridinium $p$-toluenesulfonate $(150 \mathrm{mg}, 0.5 \mathrm{mmol})$ with piperidinium $\mathrm{O}, \mathrm{O}^{\prime}$-di- $m$-tolylphosphorodithioate $\quad(\mathbf{1 g})$ (395 mg, $1 \mathrm{mmol}$ ), followed by TLC [dichloromethane/hexane (1:5), $\left.R_{f}=0.48\right]$ and by recrystallization from ether/hexane (1:3), gave $158 \mathrm{mg}(54 \%)$ of $\mathbf{3} \mathbf{g}$ as colorless crystals.

$$
\begin{aligned}
& \mathrm{C}_{28} \mathrm{H}_{28} \mathrm{P}_{2} \mathrm{O}_{4} \mathrm{~S}_{3} \text { (586.55) } \\
& \text { Found C 57.47 H 4.78, } \\
& \text { Calcd C } 57.33 \text { H } 4.81 \text {. }
\end{aligned}
$$

\section{Bis $\left(O, O^{\prime}\right.$-di-p-tolylphosphorothioyl) sulfide (3h)}

2-Chloro-1-methylpyridinium $p$-toluenesulfonate (150 mg, $0.5 \mathrm{mmol}$ ) was added to a solution of piperidinium $\mathrm{O}, \mathrm{O}^{\prime}$-di-p-tolylphosphorodithioate $(\mathbf{1} \mathbf{h})$ $(395 \mathrm{mg}, 1 \mathrm{mmol})$ in dichloromethane $(10 \mathrm{ml})$ and the reaction mixture was stirred at $0{ }^{\circ} \mathrm{C}$ for $1 \mathrm{~h}$. After removal of the solvent by rotary evaporator, thin layer chromatography of the residue (hexane/ethyl acetate $=5: 1, R_{f}=0.81$ ) followed by recrystallization of the resulting solid from hexane gave $174 \mathrm{mg}$ $(59 \%)$ of $\mathbf{3 h}$ as colorless crystals: m.p. $91-93{ }^{\circ} \mathrm{C}$ and $38 \mathrm{mg}(61 \%)$ of 1 -methylpyridine-2-thione $(4)\left(R_{f}=\right.$ $0.57)$ as yellow crystals: m.p. $81-82{ }^{\circ} \mathrm{C}$. The m.p. and IR, ${ }^{1} \mathrm{H}$ NMR, and mass spectra of $\mathbf{3} \mathbf{h}$ and $\mathbf{4}$ were consistent with those of the authentic samples, which were prepared by the reaction of $\mathrm{O}, \mathrm{O}^{\prime}$-di- $p$-tolylphosphorodithioic acid with dicyclohexylcarbodiimide [9] or of 4-methylbenzenecarbodithioic acid with 2-chloro-1-methylpyridinium iodide [13], respectively.

\section{$\operatorname{Bis}\left(O, O^{\prime}\right.$-di-2-methoxybenzenephosphorothioyl) sulfide (3i)}

The reaction of 2-chloro-1-methylpyridinium $p$-toluenesulfonate $(150 \mathrm{mg}, \quad 0.5 \mathrm{mmol})$ with piperidinium $\mathrm{O}, \mathrm{O}^{\prime}$-di-2-methoxybenzenephosphorodithioate (1i) $(427 \mathrm{mg}, 1 \mathrm{mmol})$, followed by TLC [ethyl acetate/hexane (1:2), $R_{f}=0.48$ ], gave $98 \mathrm{mg}$ $(30 \%)$ of $\mathbf{3 i}$ as pale yellow oil.

\section{$\operatorname{Bis}\left(O, O^{\prime}\right.$-di-4-methoxybenzenephosphorothioyl sulfide $(\mathbf{3} \mathbf{j})$}

The reaction of 2-chloro-1-methylpyridinium $p$-toluenesulfonate $(150 \mathrm{mg}, \quad 0.5 \mathrm{mmol})$ with piperidinium $\mathrm{O}, \mathrm{O}^{\prime}$-di-3-methoxybenzenephosphorodithioate (1j) (427 mg, $1 \mathrm{mmol}$ ), followed by TLC [ethyl acetate/hexane $(1: 2), R_{f}=0.42$ ] and by recrystallization from ether/hexane (1:1), gave $244 \mathrm{mg}$ $(75 \%)$ of $\mathbf{3} \mathbf{j}$ as colorless crystals.

$$
\begin{array}{ccc}
\mathrm{C}_{28} \mathrm{H}_{28} \mathrm{P}_{2} \mathrm{O}_{8} \mathrm{~S}_{3} & \text { (650.65) } \\
\text { Found } & \mathrm{C} 51.26 & \mathrm{H} 4.20, \\
\text { Calcd } & \mathrm{C} 51.69 & \mathrm{H} 4.34 .
\end{array}
$$

\section{Bis $\left(O, O^{\prime}\right.$-di-4-chlorobenzenephosphorothioyl) sulfide (3k)}

The reaction of 2-chloro-1-methylpyridinium $p$-toluenesulfonate $(150 \mathrm{mg}, \quad 0.5 \mathrm{mmol})$ with piperidinium $\mathrm{O}, \mathrm{O}^{\prime}$-di-4-chlorobenzenephosphorodithioate $(\mathbf{1 k})(436 \mathrm{mg}, 1 \mathrm{mmol})$, followed by TLC [dichloromethane/hexane (1:2), $\left.R_{f}=0.50\right]$ and by re- 
crystallization from ether/hexane (1:3), gave $192 \mathrm{mg}$ $(57 \%)$ of $\mathbf{3} \mathbf{k}$ as colorless crystals.

$$
\begin{gathered}
\mathrm{C}_{24} \mathrm{H}_{16} \mathrm{P}_{2} \mathrm{O}_{4} \mathrm{~S}_{3} \mathrm{Cl}_{4}(668.33) \\
\text { Found } \\
\text { Calcd }
\end{gathered}
$$

\section{Bis(diphenylphosphinothioyl) oxide (8)}

2-Chloro-1-methylpyridinium $p$-toluenesulfonate (150 mg, $0.5 \mathrm{mmol}$ ) was added to a solution of piperidinium diphenylphosphorothioate (319 $\mathrm{mg}$, $1 \mathrm{mmol})$ in dichloromethane $(10 \mathrm{ml})$ at $0{ }^{\circ} \mathrm{C}$, and the reaction mixture was stirred for $30 \mathrm{~min}$. The solvent was evaporated by rotary evaporator. TLC of the residue (hexane/ethyl acetate $=5: 1, R_{f}=0.57$ ), followed by recrystallization from dichloromethane/ hexane, gave $101 \mathrm{mg}(45 \%)$ of $\mathbf{8}$ as colorless crystals. M.p. $193-195^{\circ} \mathrm{C}$; MS $(20 \mathrm{eV}) \mathrm{m} / z 450\left[\mathrm{M}^{+}\right](100)$, $373\left[\mathrm{Ph}_{2} \mathrm{P}(\mathrm{S}) \mathrm{OP}(\mathrm{S}) \mathrm{Ph}\right]^{+}(3), 341\left[\mathrm{Ph}_{2} \mathrm{P}(\mathrm{S}) \mathrm{OPPh}\right]^{+}$ (30), $201\left[\mathrm{Ph}_{2} \mathrm{PO}\right]^{+}(10) ;{ }^{31} \mathrm{P} \mathrm{NMR}\left(\mathrm{CH}_{2} \mathrm{Cl}_{2}\right.$, reference $\left.85 \% \mathrm{H}_{3} \mathrm{PO}_{4}\right)$ 80.01; IR (KBr): $v\left(\mathrm{~cm}^{-1}\right) 2990$,
$1470,1425,1310,1270,1100,920,905,770,750,720$, $690,685,650,610,585,525,505,495,425$.

\section{Bis(diphenylphosphinothioyl) sulfide (11)}

2-Chloro-1-methylpyridinium $p$-toluenesulfonate (150 mg, $0.5 \mathrm{mmol}$ ) was added to a solution of piperidinium diphenylphosphinodithioate $(335 \mathrm{mg}$, $1 \mathrm{mmol})$ in dichloromethane $(10 \mathrm{ml})$. The reaction mixture was evaporated by rotary evaporator. TLC of the residue (hexane/ethyl acetate $=2: 1$ ) followed by recrystallization of the resulting solid from hexane gave $145 \mathrm{mg}(62 \%)$ of $\mathbf{1 1}\left(R_{f}=0.56\right)$ as colorless crystals, m.p. $121-122{ }^{\circ} \mathrm{C}$ [lit. [16] $121.5^{\circ} \mathrm{C}$ ] and $41 \mathrm{mg}(65 \%)$ of 4.

11: MS (20 eV) $m / z 466\left(\mathrm{M}^{+}\right) ;{ }^{31} \mathrm{P} \mathrm{NMR}\left(\mathrm{CH}_{2} \mathrm{Cl}_{2}\right.$, reference $\left.85 \% \quad \mathrm{H}_{3} \mathrm{PO}_{4}\right)$ 61.52; $\mathrm{IR}(\mathrm{KBr}): v\left(\mathrm{~cm}^{-1}\right)$ $3010,1800,1660,1570,1465,1420,1290,1250,1175$, $1155,1005,970,910,735,705,670,630,600,520$, $470,450,430,410,400$.
[1] Gy. Matolesy and Z. Feket, Novenytemeles [Pflanzenbau] 5, 311 (1956).

[2] R. J. Magee (American Cyanamide Co.), U.S. Patent, 3467734 (1969, Sept.).

[3] The reaction of phosphorodithioic acid salts with phosphorochlorothioate led to phosphorodithioester [(RO $\left.)_{2} \mathrm{PS}_{2} \mathrm{R}\right]:$ P. W. Vogel and N. A. Meinhardt, U.S. Patent, 2900406 (1959).

[4] L. Malatesta, Gazz. Chim. Ital. 81, 596 (1951).

[5] L. Almasi and A. Hantz, Chem. Ber. 97, 661 (1964) and literature cited therein.

[6] R. S. Edmundson, Tetrahedron 21, 2379 (1965).

[7] N. N. Mel'nikov, K. D. Shvetsova-Shilovoskaya, and M. Ya. Kagan, J. Gen. Chem. 30, 2300 (1960); C. A. 55, 9320i (1961).

[8] A. E. Lipman, J. Org. Chem. 30, 3217 (1965).
[9] M. Mikolajczyk, P. Kielbasinskii, and W. Basinski, J. Org. Chem. 49, 899 (1984).

[10] S. Kato, H. Masumoto, M. Kimura, T. Murai, and M. Ishida, Synthesis 1987, 304.

[11] H. Masumoto, S. Kato, H. Muramaru, and T. Murai, unpublished data.

[12] L. Almasi and A. Hantz, Chem. Ber. 99, 3288 (1966).

[13] I. P. Komkov and V. M. Levitskaya, Izv. Vyssh. Ucheb. Zaved. Khim. Tekhnol. 10, 1014 (1967).

[14] N. I. Zemlyanski and D. S. Drach, J. Gen. Chem. 32, 1942 (1962); C. A. 58, 4450d (1963).

[15] M. G. B. Drew, R. J. Hobson, P. E. M. Mumba, and D. A. Rice, J. Chem. Soc. Dalton Trans. 1987, 1569.

[16] S. L. Lawton and G. T. Kokotailo, Inorg. Chem. 8, 2410 (1969).

[17] L. Almasi and L. Paskucz, Chem. Ber. 102, 1489 (1962). 